\title{
Stone-Cold Low Temperature Cathodoluminescence Spectrometry of Quartz $\left(\mathrm{SiO}_{2}\right)$
}

\author{
Edward P. Vicenzi ${ }^{1,2}$ and Scott A. Wight ${ }^{2}$ \\ 1. Museum Conservation Institute, Smithsonian Institution, Suitland, MD, USA \\ 2. National Institute of Standards and Technology, Gaithersburg, MD, USA
}

Cathodoluminescence (CL) studies of quartz have been useful in Earth sciences for understanding geological cycling [1], quantitative trace element concentrations and thermal properties of crystallization [2], as well as serving as a petrographic aid to interpret mass transport properties [3]. The spectral characteristics of quartz cement were recently determined to constrain the origin of silica in sandstones being evaluated for geological storage of atmospheric $\mathrm{CO}_{2}$ [4]. The authors used liquid nitrogen cooling to reduce electron beam damage while increasing CL intensity. In this study, hyperspectral CL data for detrital and cementitious quartz have been measured at temperatures as low as $6 \mathrm{~K}$.

SEM-based CL data were collected using an FEI Apreo SEM with a $15 \mathrm{kV} / 1.6 \mathrm{nA}$ electron beam. Spectra were collected using a Gatan MonoCL4 Elite cathodoluminescence system equipped with a high sensitivity PMT, a 150 lines/mm grating, and a Princeton Instruments PIXIS 100BR CCD. Cooling was achieved by means of a Gatan CF302 liquid helium cooling stage $(6 \mathrm{~K}+/-1)$ and a model 1905 digital temperature controller with a type 6 silicon diode temperature sensor. The quartz examined is from the Ordovician St. Peter sandstone, and the specimen was provided by the University of Wisconsin as a proposed SEM-CL reference material for the microanalysis community [5].

Transported and deposited (detrital) quartz displays both high CL spectral intensity and a pronounced time dependence at $1.9 \mathrm{eV}$ (Fig. A, $\mathrm{A}_{1}, \mathrm{~A}_{2}$ ). This spectral feature has been linked to a non-bridging oxygen hole center $(\mathrm{NBOHC})$, or a $\mathrm{NBOHC}$ with a non-bridging alkali impurity in the $\alpha$-quartz $\mathrm{SiO}_{4}$ polyhedral network [6]. Despite cooling to liquid helium temperatures, the intensity of the $1.9 \mathrm{eV}$ feature increases significantly with increasing electron dose over a period of single digit seconds. Such time dependency is related to defect generation in the interaction volume, coupled with the irradiation-induced electric field produced by trapped charges at defects [7]. Conversely, signal from the quartz cement is weaker, and shows a time dependent loss of intensity of the $2.7 \mathrm{eV}$ feature (Fig. B, $\mathrm{B}_{1}$ ). Imaging across a grain boundary reveals quartz cement that appears epitaxial with one grain, yet shares spectral features with an adjacent grain (Fig. 2). A line profile across a grain boundary with a thick over layer of cement illustrates a more typical quartz cement spectrum comprised of a $2.7 \mathrm{eV}$ feature and a less significant $1.9 \mathrm{eV}$ peak $[8]$.

References:

[1] J Screiber et al., Nature 406 (2000), p 981.

[2] WP Leeman et al., Microsc. Microanal. 18 (2012), p. 1322.

[3] KL Milliken in “Treatise on Geochemistry, Volume 7" Ed: FT Mackenzie, (Elsevier), p. 159.

[4] CM MacRae et al., IOP Conf. Ser.: Mater. Sci. Eng. 304 (2018), 14 p.

[5] J Fournelle, Microanalytical Reference Materials Conference, (2012).

[6] MA Stevens and MR Phillips, Phys. Rev. B. 52 (1995), p 3122.

[7] MA Stevens, Min. Mag. 73 (2009), p 585.

[8] Certain commercial equipment, instruments, or materials are identified in this report to specify adequately the experimental procedure. Such identification does not imply recommendation or 
endorsement by the National Institute of Standards and Technology, nor does it imply that the materials or equipment identified are necessarily the best available for the purpose.

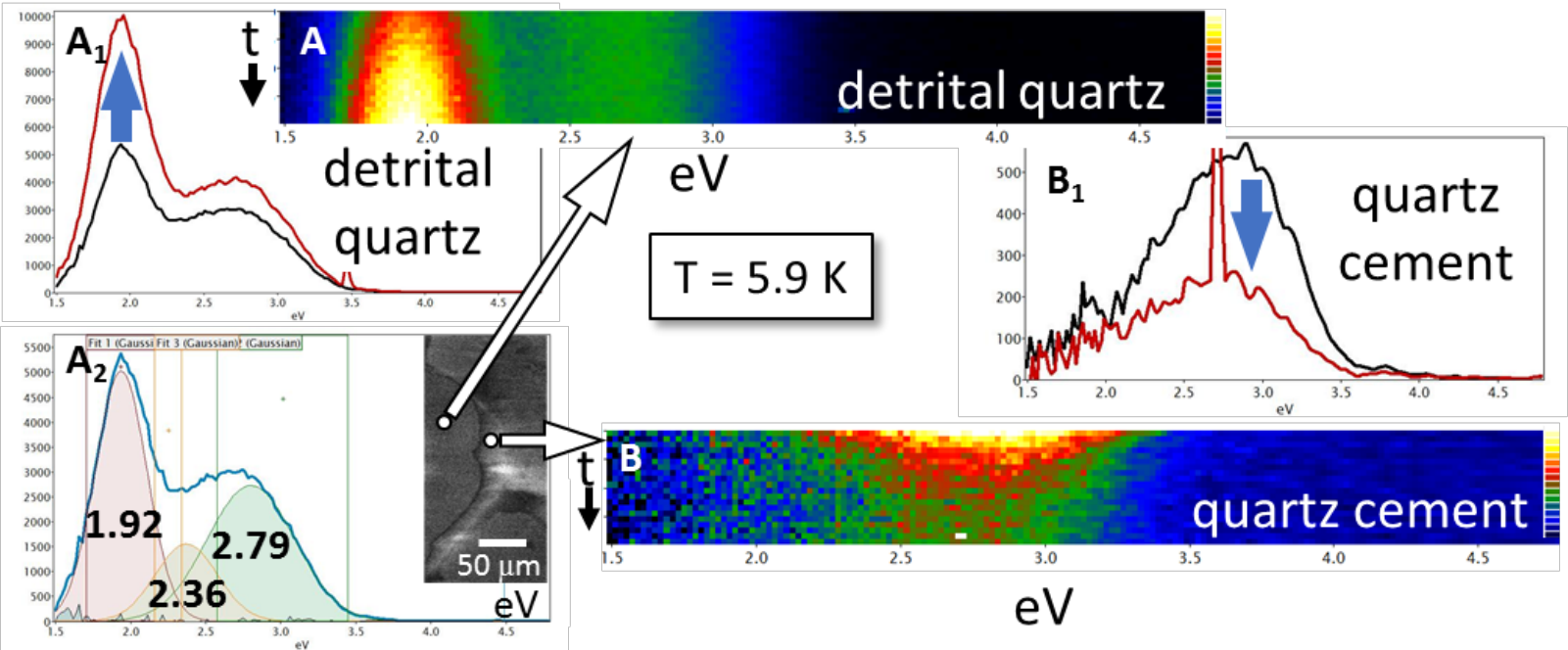

Figure 1. $\mathrm{CL}$ time series spectra $(20 \times 400 \mathrm{~ms})$ conducted at $5.9 \mathrm{~K}$. A) Spectral time dependence for detrital quartz. $A_{1}$ ) Sum of initial 3 (black), and final 3 (red) time steps. $A_{2}$ ) Gaussian peak fits, centroids, and residuals for the black spectrum in $A_{1}$. Inset: panchromatic image showing location of time series spectra. B) Spectral time dependence for quartz cement. $B_{1}$ ) Sum of initial 3 (black), and final 3 (red) time steps.
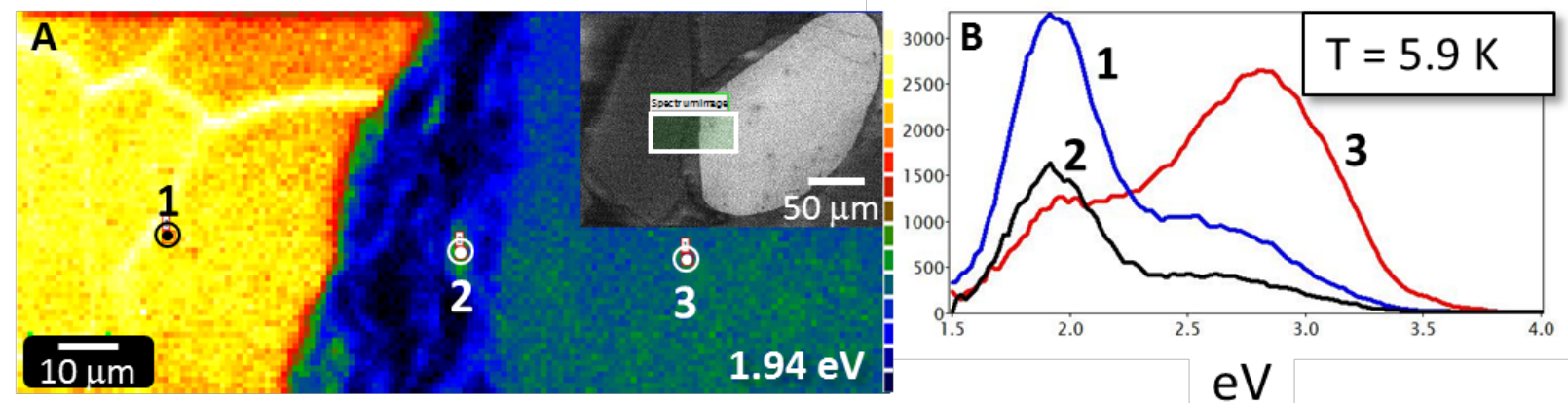

Figure 2. $\mathrm{CL}$ spectrum image collected at $5.9 \mathrm{~K}$ of detrital quartz grain boundary with quartz cement interface. A) $1.94 \mathrm{eV}$ image slice. Inset: lower magnification panchromatic image with white rectangle highlighting the $\mathrm{CL}$ spectrum image. B) 3 derived $\mathrm{CL}$ spectra from locations marked on $2 \mathrm{~A}$.
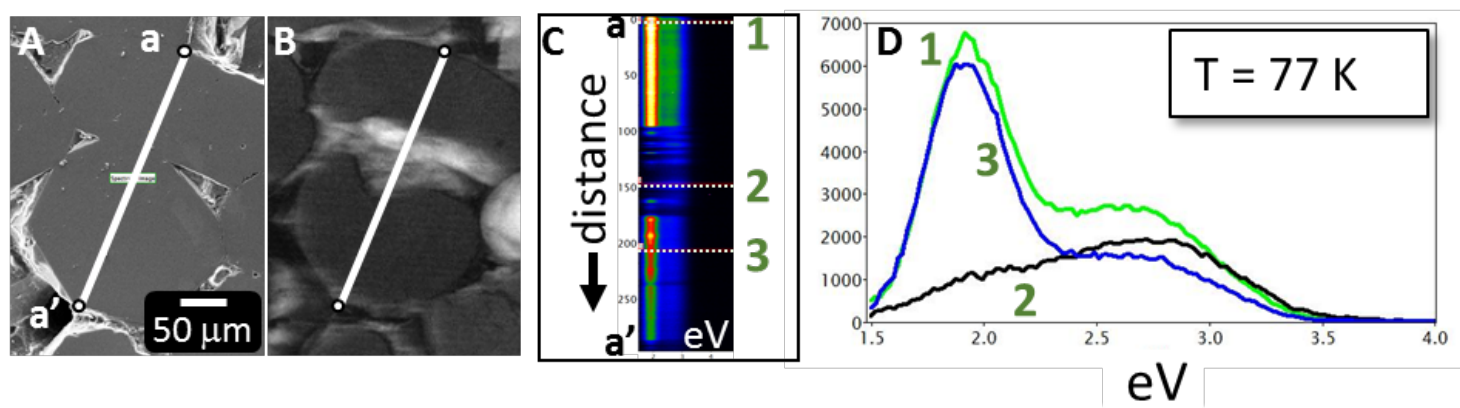

Figure 3. CL spectrum line profile collected at $77 \mathrm{~K}$ across 2 detrital quartz grains sutured by a thick zone of quartz cement. $\mathrm{A}, \mathrm{B}$ ) secondary electron and panchromatic $\mathrm{CL}$ images with dashed lines marking the spectrum profile from a-a'. C) 1D CL spectrum profile. D) 3 derived CL spectra from positions marked on $3 C$. 